\title{
Incorporation of a sequential BMP-2/BMP-7 delivery system into chitosan-based scaffolds for bone tissue engineering
}

\author{
Pinar Yilgor ${ }^{a}$, Kadriye Tuzlakoglu ${ }^{\mathrm{b}}$, Rui L. Reis ${ }^{\mathrm{b}}$, Nesrin Hasirci ${ }^{\text {a,c,d }}$, Vasif Hasirci ${ }^{\text {a, }}$,e,* \\ ${ }^{a}$ METU, BIOMAT, Department of Biotechnology, 06531 Ankara, Turkey \\ b 3B's Research Group - Biomaterials, Biodegradables and Biomimetics, IBB-Institute for Biotechnology and Bioengineering, PT Associated Laboratory, University of Minho, \\ Headquarters of the European Institute of Excellence on Tissue Engineering and Regenerative Medicine, AvePark, 4806-909 Taipas, Guimarães, Portugal \\ ${ }^{\mathrm{c}}$ METU, BIOMAT, Department of Chemistry, 06531 Ankara, Turkey \\ ${ }^{\mathrm{d}}$ METU, BIOMAT, Department of Biomedical Engineering, 06531 Ankara, Turkey \\ ${ }^{\mathrm{e}}$ METU, BIOMAT, Department of Biological Sciences, Biotechnology Research Unit, 06531 Ankara, Turkey
}

\section{A R T I C L E I N F O}

\section{Article history:}

Received 8 January 2009

Accepted 11 March 2009

Available online 9 April 2009

\section{Keywords:}

Bone tissue engineering

Sequential delivery

BMP

PLGA

PHBV

Chitosan

\begin{abstract}
A B S T R A C T
The aim of this study was to develop a 3-D construct carrying an inherent sequential growth factor delivery system. Poly(lactic acid-co-glycolic acid) (PLGA) nanocapsules loaded with bone morphogenetic protein BMP-2 and poly(3-hydroxybutyrate-co-3-hydroxyvalerate) (PHBV) nanocapsules loaded with BMP-7 made the early release of BMP-2 and longer term release of BMP-7 possible. 3-D fiber mesh scaffolds were prepared from chitosan and from chitosan-PEO by wet spinning. Chitosan of $4 \%$ concentration in $2 \%$ acetic acid (CHI4-HAc2) and chitosan (4\%) and PEO (2\%) in 5\% acetic acid (CHI4PEO2-HAc5) yielded scaffolds with smooth and rough fiber surfaces, respectively. These scaffolds were seeded with rat bone marrow mesenchymal stem cells (MSCs). When there were no nanoparticles the initial differentiation rate was higher on (CHI4-HAc2) scaffolds but by three weeks both the scaffolds had similar alkaline phosphatase (ALP) levels. The cell numbers were also comparable by the end of the third week. Incorporation of nanoparticles into the scaffolds was achieved by two different methods: incorporation within the scaffold fibers (NP-IN) and on the fibers (NP-ON). It was shown that incorporation on the $\mathrm{CHI} 4-\mathrm{HAc} 2$ fibers (NP-ON) prevented the burst release observed with the free nanoparticles, but this did not influence the total amount released in 25 days. However NP-IN for the same fibers revealed a much slower rate of release; ca. $70 \%$ released at the end of incubation period. The effect of single, simultaneous and sequential delivery of BMP-2 and BMP-7 from the CHI4-HAc2 scaffolds was studied in vitro using samples prepared with both incorporation methods. The effect of delivered agents was higher with the NP-ON samples. Delivery of BMP-2 alone suppressed cell proliferation while providing higher ALP activity compared to BMP-7. Simultaneous delivery was not particularly effective on cell numbers and ALP activity. The sequential delivery of BMP-2 and BMP-7, on the other hand, led to the highest ALP activity per cell (while suppressing proliferation) indicating the synergistic effect of using both growth factors holds promise for the production of tissue engineered bone.
\end{abstract}

(c) 2009 Elsevier Ltd. All rights reserved.

\section{Introduction}

The limited ability of bone tissue to regenerate in case of large defects created the need for substitutes which are mostly of autogenic and allogenic origin [1]. Tissue engineered constructs emerged as promising alternatives to these grafts to form viable and functional 3-D constructs. Polymeric foams [2], micro and/or

\footnotetext{
* Corresponding author. BIOMAT, Middle East Technical University, Department of Biological Sciences, Biotechnology Research Unit, Inonu Bulvari, 06531 Ankara, Turkey, Tel.: +90 312210 5180; fax: +90 3122101542

E-mail address: vhasirci@metu.edu.tr (V. Hasirci).
}

nanofiber-based scaffolds [3-5] and rapid prototyped constructs $[6,7]$ are among the structures that have been successfully employed as scaffolds for bone tissue engineering; however, control of cell activity, especially differentiation, within the scaffold has not been fully achieved in these systems.

Growth factors regulate cellular activities in vivo and their application as external bioactive agents has been reported to enhance bone healing $[8,9]$, control growth and differentiation of cells [10] and stimulate angiogenesis [11]. In nature, multiple growth factors such as bone morphogenetic proteins (BMPs), insulin-like growth factor (IGF), fibroblast growth factor (FGF) and vascular endothelial growth factor (VEGF) function in unison during bone formation and fracture healing processes [12]. 
Among these, BMPs were shown to induce bone formation by inducing mesenchymal stem cells (MSCs) toward chondroblastic and osteoblastic differentiation [13]. BMP-2 and BMP-7 were shown to be the most effective ones that induce complete bone morphogenesis [14] and were approved by FDA for clinical applications [15,16]. Considering their mechanism of action, BMP2 was reported to be an early appearing factor, peaking at day 1 after fracture, while BMP-7 was expressed approximately after 2 weeks [17]. Therefore, it was considered that delivery of combinations of these growth factors is a viable biomimetic approach towards bone healing.

The conventional strategy in growth factor therapy for orthopedic applications is to administer the agent in the form of a large dose by either single or repeated injections but in such applications a considerable proportion of the agent was reported to be lost through leakage and/or loss of bioactivity [12]. Encapsulation of growth factors in carrier structures, therefore, could be of utmost importance in protecting the bioactivity of the agent and prolonging its presence at the defect site. There are various recent attempts in the literature to incorporate growth factors into scaffold structures in order to provide the necessary protection and prolongation of activity as well as to achieve proper control of cellular activities in the implant side [18-20].

The most recent developments observed in the preparation of scaffold/controlled delivery systems involve the combined delivery of several growth factors from the same scaffold. The few studies in the literature include BMP-2 and transforming growth factor- $\beta 3$ (TGF- $\beta 3$ ) delivery from alginate hydrogels transplanted in mice which revealed significant healing [21]. Dual delivery of VEGF and BMP-2 from gelatin microparticles embedded in porous degradable scaffolds had a positive effect on repair of a rat cranial defect [22]. Similarly, sequential delivery of BMP-2 and then IGF-1 from two-layered gelatin coatings led to elevated alkaline phosphatase (ALP) activity and mineralized matrix formation in vitro [23].

The most recent study in the literature on the combined delivery of BMP-2 and BMP-7 was reported by our research group where the growth factors were released from complexed microspheres embedded in porous poly(lactic acid-co-glycolic acid) (PLGA) scaffolds [24]. The positive effect of co-administration of BMP-2 and BMP-7 on osteogenic differentiation was shown in vitro. In the only other study also from our group [25], a nano-scale controlled release system was developed to enable the sequential delivery of first BMP-2 and then BMP-7 from PLGA and poly(3-hydroxybutyrate-co-3-hydroxyvalerate) (PHBV) nanocapsules, respectively. The effect of various nanoparticle production parameters was investigated in order to achieve the proper release rates to constitute the parts of the sequential delivery system. Nanocapsules of PLGA and PHBV were found to have sufficiently high encapsulation efficiencies, appropriate release rates and smooth surfaces. This sequential BMP-2/BMP-7 delivery system enhanced the differentiation of MSCs into osteoblasts in vitro while decreasing the proliferation rate.

In the present study, the above mentioned nanoparticulate sequential delivery system was incorporated into and onto the fibers of wet spun chitosan-based scaffolds to create bi-functional constructs serving both as a scaffold and also as the growth factor delivery system. In the previous study [25], the unentrapped (free) nanocapsules were used to release both the bovine serum albumin (BSA) and the BMPs and were found to have similar kinetics, so in the present study the fiber-incorporated nanocapsules were expected to behave as before, presenting a similar release kinetics for the BMPs and the BSA. Therefore, in the current study only the release kinetics of BSA was studied.
Fibrous scaffolds have gained great attention over the last years as they have appropriate porosity for cell penetration, nutrient exchange and tissue ingrowth. These fibrous structures were produced by electrospinning [4], wet spinning [5] and fiber bonding [26]. In this study, chitosan fiber mesh scaffolds were used to house the nanoparticulate sequential growth factor delivery system. The effect of single, simultaneous and sequential delivery of BMP-2 and BMP-7 incorporated to the scaffolds by two different techniques, into and onto chitosan fibers, were studied in vitro using rat bone marrow MSCs.

\section{Materials and methods}

\subsection{Materials}

Low molecular weight chitosan (deacetylation degree $90.85 \%$, i.v. 185 cps for $1 \%$ in $1 \%$ acetic acid) was purchased from Aldrich. Poly(ethyleneoxide) (PEO) (Polyox ${ }^{\mathrm{TM}}$ WSR 301, $\mathrm{M}_{\mathrm{W}} 4 \times 10^{6}$ ) was obtained from Dow Chemical Company (USA). PLGA (50:50) (Resomer ${ }^{\circledR}$ RG503H, i.v. 0.32-0.44 dL/g, for $0.1 \%$ in chloroform, $25^{\circ} \mathrm{C}$ ) was purchased from Boehringer Ingelheim (Germany). PHBV (PHV content $8 \% \mathrm{w} / \mathrm{w}$ ), dexamethasone, $\beta$-glycerophosphate disodium salt, $\mathrm{L}$-ascorbic acid were bought from Sigma-Aldrich (Germany). BSA and polyvinyl alcohol (PVA) ( $\mathrm{M}_{\mathrm{W}}$ 15,000) were from Fluka (USA). BMP-2 from InductOs kit (Medtronic, USA) and recombinant human BMP-7 from Ray Biotech (USA) were used as the growth factors. Quantikine BMP-2 immunoassay from R\&D Systems (USA) and human BMP-7 Elisa kit from Ray Biotech (USA) were used in the determination of the growth factors. Dulbecco's Modified Eagle Medium (DMEM, high glucose), fetal bovine serum (FBS) were obtained from Hyclone (USA). NucleoCounter reagents were supplied by Chemometec (Denmark) and Alamar Blue cell proliferation assay was from USBiological. For the assessment of cell differentiation, alkaline phosphatase kit (Randox, USA) was used.

\subsection{Preparation of BSA, BMP-2 and BMP-7 loaded nanocapsules}

PLGA and PHBV nanocapsules containing BMP-2 and BMP-7, respectively, or BSA in both type of nanocapsules, were prepared by the double emulsion-solvent evaporation technique as reported earlier [25]. Briefly, an aqueous solution of BSA or BMP was emulsified in dichloromethane containing PLGA or PHBV and this was then introduced to an aqueous solution containing PVA. Nanocapsules were collected by centrifugation and washed with Tris- $\mathrm{HCl}(\mathrm{pH} 7.4)$. The nanocapsules were then resuspended in the buffer and lyophilized.

\subsection{Chitosan-based fiber mesh scaffold production}

Chitosan-based 3-D fiber mesh scaffolds were prepared by wet spinning of chitosan and chitosan/PEO blends according to the procedure described before [3]. Briefly, chitosan and chitosan/PEO were dissolved in aqueous acetic acid. These solutions were then injected into a coagulation bath of $\mathrm{Na}_{2} \mathrm{SO}_{4}(0.5 \mathrm{M}), \mathrm{NaOH}(1 \mathrm{~m})$ and distilled water $(3: 1: 6 \mathrm{v} / \mathrm{v})$ in which they were kept overnight. After exhaustive washing with distilled water, fibers were dehydrated with methanol and then dried in a mould. Chitosan was blended with PEO to study its effect on the mechanical and structural properties of the construct. The effect of chitosan concentration, PEO addition and acetic acid concentration were studied by preparation of the samples listed in Table 1.

\subsection{Micro-computed tomography}

Mean porosity and porosity distribution of the 3-D scaffolds were assessed by using micro-computed tomography ( $\mu$-CT 20, SCANCO Medicals, Switzerland). Scanner settings were $40 \mathrm{keV}$ and $248 \mu \mathrm{A}$. Entire scaffolds were scanned in slices of $7 \mu \mathrm{m}$ thickness. CT Analyser and CT Vol Realistic 3-D Visualization (SkyScan, Belgium) softwares were used for image processing in CT reconstructions, and in creation and visualization of the 3-D representations.

Table 1

Components of chitosan-based scaffolds and their compositions.

\begin{tabular}{lllll}
\hline Sample code & $\begin{array}{l}\text { Surface } \\
\text { topography }\end{array}$ & \multicolumn{3}{l}{ Concentration in final solution } \\
\cline { 3 - 5 } & & $\begin{array}{l}\text { Chitosan } \\
(\%)(\mathrm{w} / \mathrm{v})\end{array}$ & $\begin{array}{l}\text { PEO } \\
(\%)(\mathrm{w} / \mathrm{v})\end{array}$ & $\begin{array}{l}\text { Acetic } \\
\text { acid }(\%)(\mathrm{v} / \mathrm{v})\end{array}$ \\
\hline CHI4-HAc2 & Smooth & 4 & - & 2 \\
CHI6-HAc2 & Rough & 6 & - & 2 \\
CHI4-PEO2-HAc2 & Smooth & 4 & 2 & 2 \\
CHI4-PEO2-HAc5 & Rough & 4 & 2 & 5 \\
\hline
\end{tabular}




\subsection{Incorporation of the nanoparticles into chitosan scaffolds}

Incorporation of PLGA and PHBV nanocapsules into chitosan-based fiber mesh scaffolds was carried out by two methods: incorporation within the fibers (NP-IN) or on the fibers (NP-ON). For incorporation within the fibers (NP-IN), particles were mixed with chitosan or chitosan/PEO solutions before the wet spinning process $\mathrm{NP}-\mathrm{ON}$ particle loading was done by introducing $100 \mu \mathrm{L}$ of nanocapsule suspension onto the both sides of the scaffolds and applying a series of vacuum-pressure cycles. The scaffolds were then dried overnight under vacuum and stored in a desiccator For the BMP loaded particle incorporated scaffolds, $40 \mathrm{ng} \mathrm{BMP} /$ scaffold was used for all conditions. For both NP-IN and NP-ON cases, single delivery of BMP-2 and BMP-7 was achieved by their encapsulation within PLGA and PHBV nanocapsules, respectively, and by their single incorporation to the constructs. For the simultaneous case, both BMP-2 and BMP-7 were encapsulated in PLGA nanocapsules, therefore performing the rapid release of both BMPs from the constructs. The sequential delivery, on the other hand, was achieved by encapsulation of BMP-2 in PLGA and BMP-7 in PHBV nanocapsules and thus achieving two different release rates.

\subsection{In situ release studies}

Release from the nanoparticle incorporated constructs was simulated by using BSA as a model molecule to represent growth factors. Protein release was determined spectrophotometrically by using Coomassie Plus Assay (Pierce, USA).

\subsection{Cell culture}

Bone marrow MSCs were isolated from 6 weeks old, male Sprague-Dawley rats as reported earlier [27]. The rats were euthanized and their femurs and tibia were excised, washed with DMEM containing $1000 \mathrm{U} / \mathrm{mL}$ penicillin and $1000 \mu \mathrm{g}$ $\mathrm{mL}$ streptomycin under aseptic conditions. The marrow in the midshaft was flushed out with DMEM containing 20\% FBS and $100 \mathrm{U} / \mathrm{mL}$ penicillin and $100 \mu \mathrm{g} /$ $\mathrm{mL}$ streptomycin, the cells were centrifuged at $500 \mathrm{~g}$ for $5 \mathrm{~min}$, and the resulting cell pellet was resuspended and plated in T-75 flasks. These primary cultures were incubated for 2 days. The hematopoietic and other unattached cells were removed from the flasks by repeated washes with phosphate buffered saline (PBS) (10 mm, $\mathrm{pH}$ 7.4) and the medium of the flasks was renewed every other day until confluency was reached. These primary cultures were then stored frozen in liquid nitrogen until use. Ethylene oxide (EtO) sterilized fiber mesh scaffolds and nanoparticle incorporated constructs were then seeded with these cells at a seeding density of 50,000 cells/scaffold. The viable cell number during cell seeding was determined with the Nucleocounter (Chemometec, Denmark). Incubation was performed at $37{ }^{\circ} \mathrm{C}$ and $5 \% \mathrm{CO}_{2}$ in DMEM supplemented with $10 \%$ FBS, $10 \mathrm{~mm} \beta$-glycerophosphate, $50 \mu \mathrm{g} / \mathrm{mL}$ L-ascorbic acid, $10 \mathrm{~nm}$ dexamethasone and penicillin/streptomycin/amphotericin B. Viable cell number was assessed with Alamar Blue assay (USBiological). ALP activity was determined by using Randox kit (USA) where the absorbance of $p$-nitrophenol formed from $p$-nitrophenyl phosphate was measured at $405 \mathrm{~nm}$.

\subsection{Scanning electron microscopy}

The structure of the scaffolds, nanocapsule incorporated constructs and the cell attachment on the fiber surfaces after 21 days of incubation were studied by Scanning Electron Microscopy (SEM) after sputter coating with gold (Leica Cambridge S360, Germany). Cell seeded scaffolds were fixed after 21 days of incubation with glutaraldehyde (2.5\% in cacodylate buffer, $\mathrm{pH} 7.4$ ) for $2 \mathrm{~h}$ and then washed with cacodylate buffer several times and lyophilized prior to SEM examination.

\subsection{Statistical analysis}

The data from the MSC proliferation and differentiation assays $(n=3)$ were analyzed with statistically significant values defined as $p<0.05$ based on one-way analysis of variance (ANOVA) followed by Tukey's test for determination of the significance of difference between different groups ( $p \leq 0.05$ ).

\section{Results and discussion}

\subsection{Wet-spun chitosan-based fiber mesh production}

Chitosan-based 3-D fiber mesh scaffolds were produced by wet spinning. The effect of polymer (chitosan) concentration, composition (chitosan, PEO) and the solvent (acetic acid) concentration on the properties of fibers were studied.

Initially, the effect of concentration of pure chitosan on the structure of the fibers produced was investigated by using 4\% (CHI4HAc2) and 6\% (CHI6-HAc2) chitosan solutions. Chitosan solutions less concentrated than $4 \%$ did not allow the formation of fibrous structures. It was observed that $4 \%$ chitosan solution was the best in terms of ease of fiber production and fiber surface smoothness whereas use of $6 \%$ chitosan gave rough surfaced fibers (Fig. 1). $8 \%$ chitosan did not even allow the formation of proper fibrous structure.

After the selection of $4 \%$ chitosan solution as the optimal concentration to prepare smooth fibers, it was further modified by blending with PEO in order to improve the structural properties. Chitosan (4\%) was blended with PEO at 2:1 ratio, creating chitosan (4\%)/PEO (2\%) fibers (CHI4-PEO2-HAc2) (Fig. 2). It was observed that introduction of $\mathrm{PEO}$ resulted in improved stability of the scaffold. However, addition of PEO more concentrated than $2 \%$ created solutions that were too viscous to be spun into proper fibers. The effect of PEO presence could be observed by comparing Figs. $1 \mathrm{~b}$ and $2 \mathrm{~b}$ in which chitosan (4\%) and
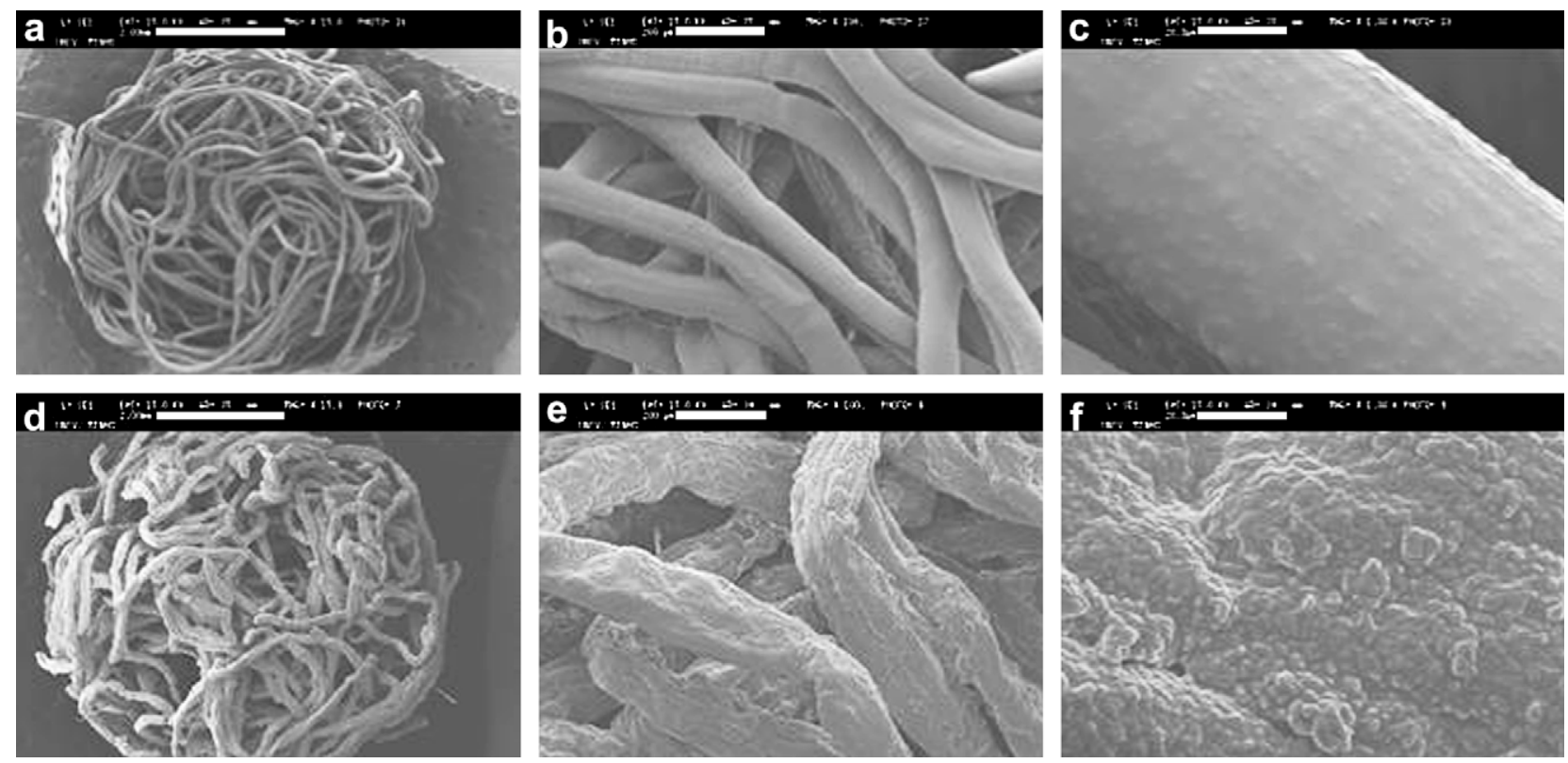

Fig. 1. CHI4-HAc2 fiber mesh scaffold, (a) $\times 15$, (b) $\times 100$, (c) $\times 1000$; CHI6-HAc2 fiber mesh scaffold, (d) $\times 15$, (e) $\times 100$, (f) $\times 1000$. Bar represents (a,d) 2 mm, (b,e) $200 \mu$ m, (c,f) $20 \mu$ m. 

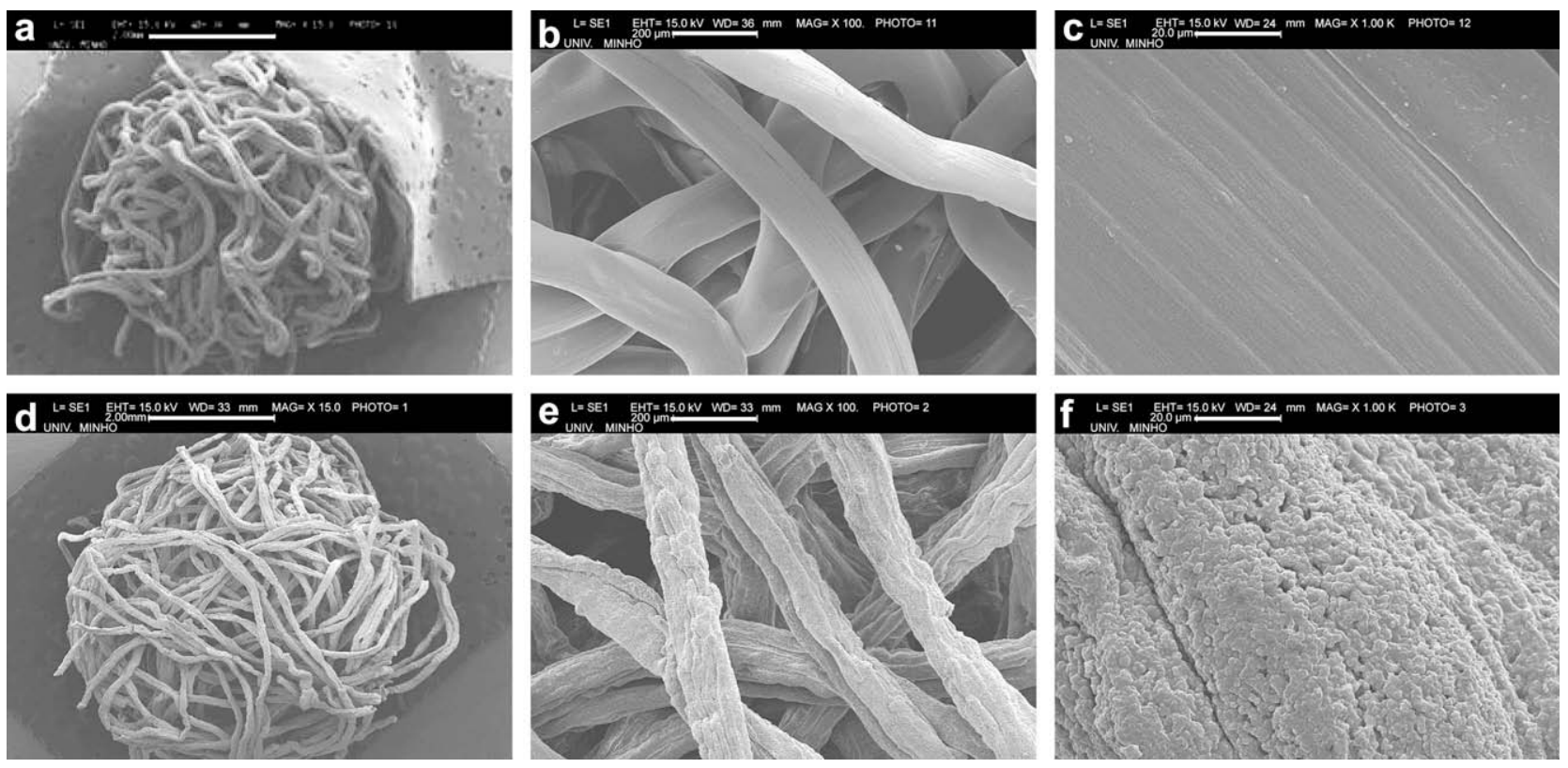

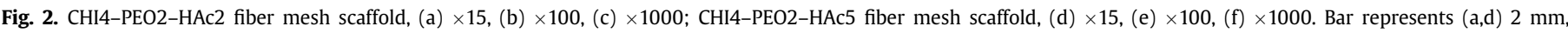
(b,e) $200 \mu \mathrm{m}$, (c, f) $20 \mu \mathrm{m}$.

chitosan (4\%)/PEO (2\%) fibers produced in $2 \%$ acetic acid are presented. PEO did not alter the smoothness of the fiber surfaces; however, resulted in increased fiber thickness, as expected due to increased total polymer concentration (ca. $100 \mu \mathrm{m}$ vs. ca. $125 \mu \mathrm{m}$ ).

Meanwhile, the effect of concentration of solvent on surface topography of the chitosan (4\%)/PEO (2\%) fibers was studied by using $2 \%$ and $5 \%(\mathrm{v} / \mathrm{v})$ acetic acid. It was observed that the more dilute acetic acid solution leads to smoother surfaces (Fig. 2).

Among the samples investigated, two scaffolds one with smooth (CHI4-HAc2) and one with rough (CHI4-PEO2-HAc5) fiber surface were selected for further investigation as the surface properties are known to make a difference in cell-material interactions through altered surface chemistry and roughness (Table 1 ). Scaffolds of CHI4-HAc2 with a smooth fiber surface, and CHI4-PEO2-HAc5 with a rough surface were used in the incorporation of the sequential delivery system.

\subsection{Characterization of the scaffolds}

The porosity and porosity distribution throughout the thickness of the scaffolds were investigated by $\mu-C T$. Analysis revealed that the

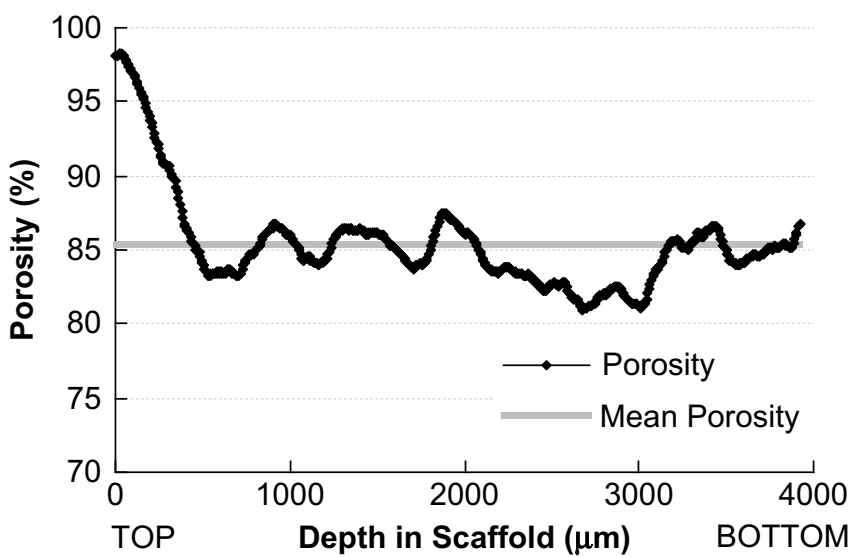

Fig. 3. Porosity distribution throughout the thickness of CHI4-HAc2 scaffold. porosity of the scaffolds was not influenced significantly by the chitosan and chitosan/PEO concentration and composition. The porosity values for CHI4-HAc2 and CHI4-PEO2-HAc5 scaffolds were $85.4 \%$ and $86.7 \%$, respectively. The porosity distribution throughout the thickness of CHI4-HAc2 scaffold is presented in Fig. 3. It was observed that the pores were completely interconnected throughout the whole structure. Moreover, the porosity profile showed that the porosity from top to bottom of the scaffold did not have a significant change. The outermost part of the scaffold has higher porosity (ca. $98 \%$ ) which reduces to ca. $85 \%$ through the bottom. This is especially important when most scaffolds produced by other methods do not have complete connectivity and the porosity decreases significantly from the surface towards the core leading to insufficient population and oxygen/nutrient concentrations in the core.

The change in properties for $\mathrm{CHI} 4-\mathrm{HAc} 2$ and $\mathrm{CHI} 4-\mathrm{PEO} 2-\mathrm{HAc} 5$ scaffolds in wet state during 21 days was investigated in sterile PBS (pH 7.4) at $37{ }^{\circ} \mathrm{C}$ and $5 \% \mathrm{CO}_{2}$ conditions. After 21 days of incubation in the medium, the dimensions and the weight of the fibers were altered significantly (Table 2 ). Both scaffolds swelled as soon as they were put into the medium and became 500-600\% (w/w) heavier. The increase in individual fiber thickness was also significant; around 55\% for both scaffolds. The diameter and height of both scaffolds were also increased, by about $20 \%$ for both of them.

The porosity of the scaffolds was measured before hydration using a $\mu$-CT. In the hydrated state the porosity was calculated (from the scaffold volume and the fiber thicknesses) to be decreased by about $15 \%$ for CHI4-HAc2 and $25 \%$ for CHI4-PEO2-HAc5. These changes are much smaller than the increase in the fiber thicknesses upon hydration (ca. 55\%) possibly because of the restraint imposed by the meshwork of the scaffold.

Table 2

Changes in chitosan-based scaffold properties after incubation in sterile PBS (pH 7.4) for 21 days.

\begin{tabular}{lcc}
\hline & CHI4-HAc2 & CHI4-PEO2-HAc5 \\
\hline Fiber thickness (\%) & 53 & 59 \\
Scaffold diameter (\%) & 18 & 29 \\
Scaffold height (\%) & 25 & 29 \\
Scaffold weight (\%) & 600 & 500 \\
\hline
\end{tabular}



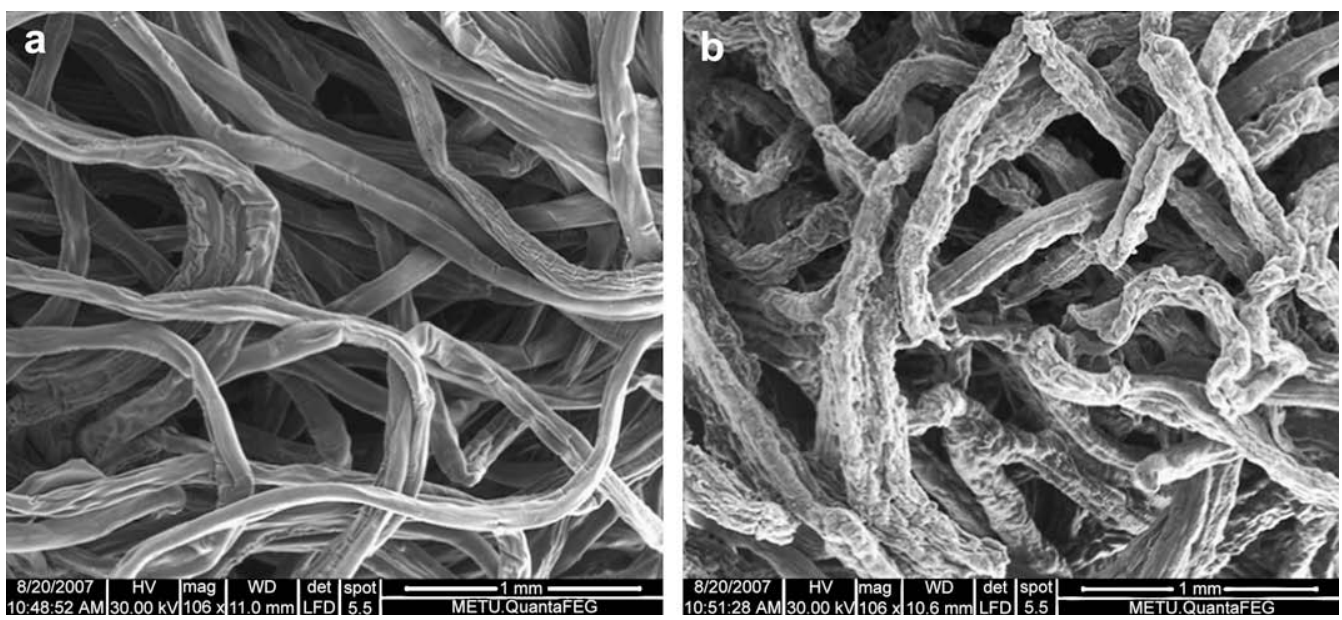

Fig. 4. Fiber structure after 21 days of incubation for (a) CHI4-HAc2, (b) CHI4-PEO2-HAc5 scaffolds.

SEM was done to examine the changes in surface properties for both scaffolds after 21 days of incubation in situ. Micrographs of CHI4-HAc2 scaffolds revealed that there was almost no change in the fiber appearance at the end of 21 days of incubation; however, PEO had dissolved out of CHI4-PEO2-HAc5 scaffolds leading to increased surface roughness (Fig. 4).

\subsection{MSC culture on chitosan-based fiber mesh scaffolds}

Suitability of chitosan-based fiber mesh scaffolds for potential use in bone tissue engineering applications were studied using rat bone marrow MSCs. It was observed that although cell numbers were almost the same after 21 days, initial cell proliferation rate during the first week was higher on CHI4-HAc2 scaffolds then on CHI4-PEO2-HAc5 (Fig. 5) which was followed by a lag period for CHI4-HAc2 scaffolds. In this lag period of cell proliferation between 7 and 14 days, the increase in ALP activity, the indicator for MSC differentiation, was higher for $\mathrm{CHI} 4-\mathrm{HAc} 2$ when compared to CHI4-PEO2-HAc5 scaffolds and the difference was statistically significant at all time points $(p<0.01)$ (Fig. 6). The change in cell proliferation was statistically significant after the first week of incubation for CHI4-HAc2 scaffolds $(p<0.05)$. Cell proliferation and ALP activity were shown to increase gradually during 21 days of incubation for blend scaffolds. ALP activities were reported as specific activity, the ALP activity per cell.

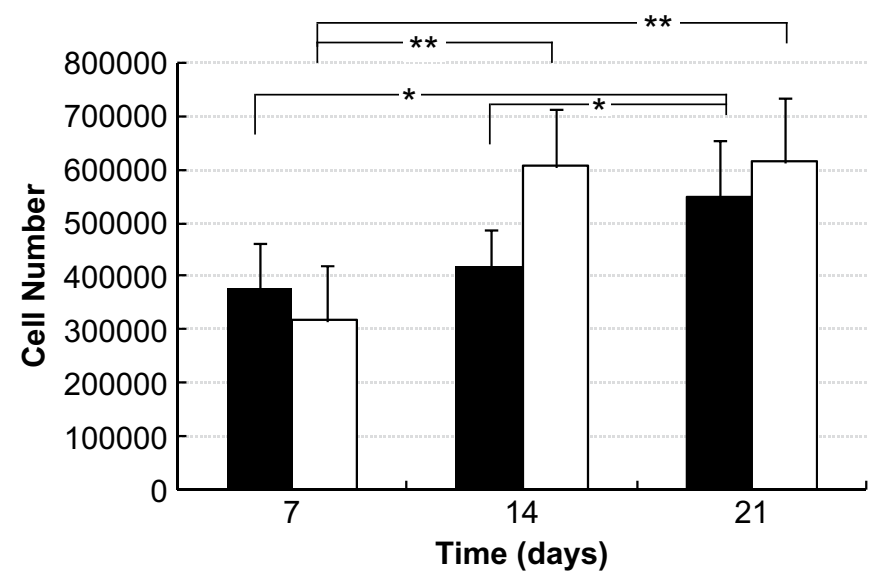

Fig. 5. MSC proliferation on CHI4-HAc2 ( $\boldsymbol{\square}$ ) and CHI4-PEO2-HAc5 ( $\square$ ) scaffolds. $\left(n=3,{ }^{*} p<0.05,{ }^{* *} p<0.001\right)$.
SEM analysis revealed that cells attached and spread well on both scaffolds after 21 days of incubation (Fig. 7). It is seen that the smooth chitosan fibers became rougher in case of cell presence after 21 days and the shapes of the cells indicate a proper spread. On CHI4-PEO2-HAc5 fibers the roughness is maintained and the cell spread is very well.

\subsection{Incorporation of sequential delivery system into chitosan-based scaffolds}

In order to incorporate the nanoparticles into chitosan-based scaffolds, two different approaches were used: nanoparticles were introduced within the chitosan-based fibers (NP-IN) or nanoparticles were incorporated onto the fibers (NP-ON).

The nanoparticles were introduced into the chitosan-based fibers by mixing the nanoparticles with chitosan and chitosan/PEO solutions followed by spinning to produce fibers containing the nanoparticles within their structure. PLGA nanocapsules incorporated into $\mathrm{CHI} 4-\mathrm{HAc} 2$ scaffolds are clearly visible due to increased roughness as unloaded fiber surfaces were smooth. The observation is difficult in CHI4-PEO2-HAc5 fibers, however, as unloaded fibers were also rough (Fig. 8).

In the second method, particles were seeded onto the CHI4HAc2 scaffolds after the preparation of both the fibers and the nanocapsules leading to the attachment of the particles onto the fiber surfaces (Fig. 9). Here the particles appear to have adhered properly onto the fibers. In this incorporation method (NP-ON),

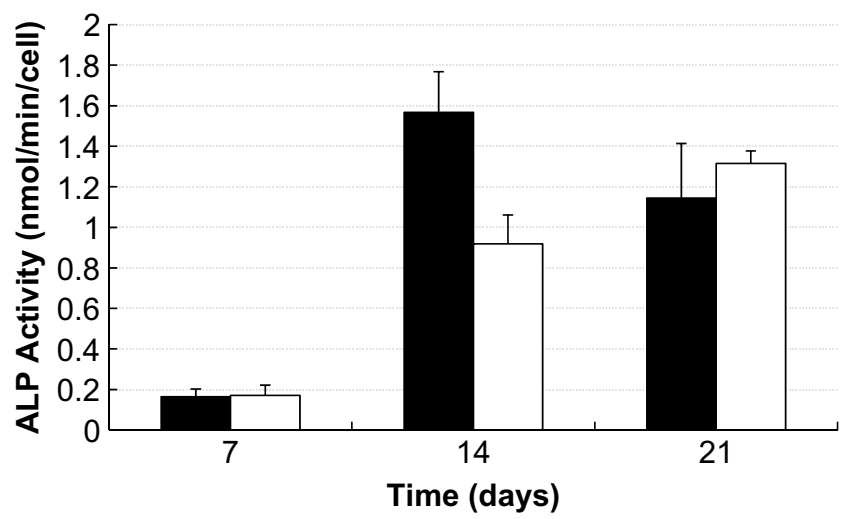

Fig. 6. Specific ALP activity on CHI4-HAc2 ( $\boldsymbol{\square}$ ) and CHI4-PEO2-HAc5 ( $\square$ ) scaffolds. 

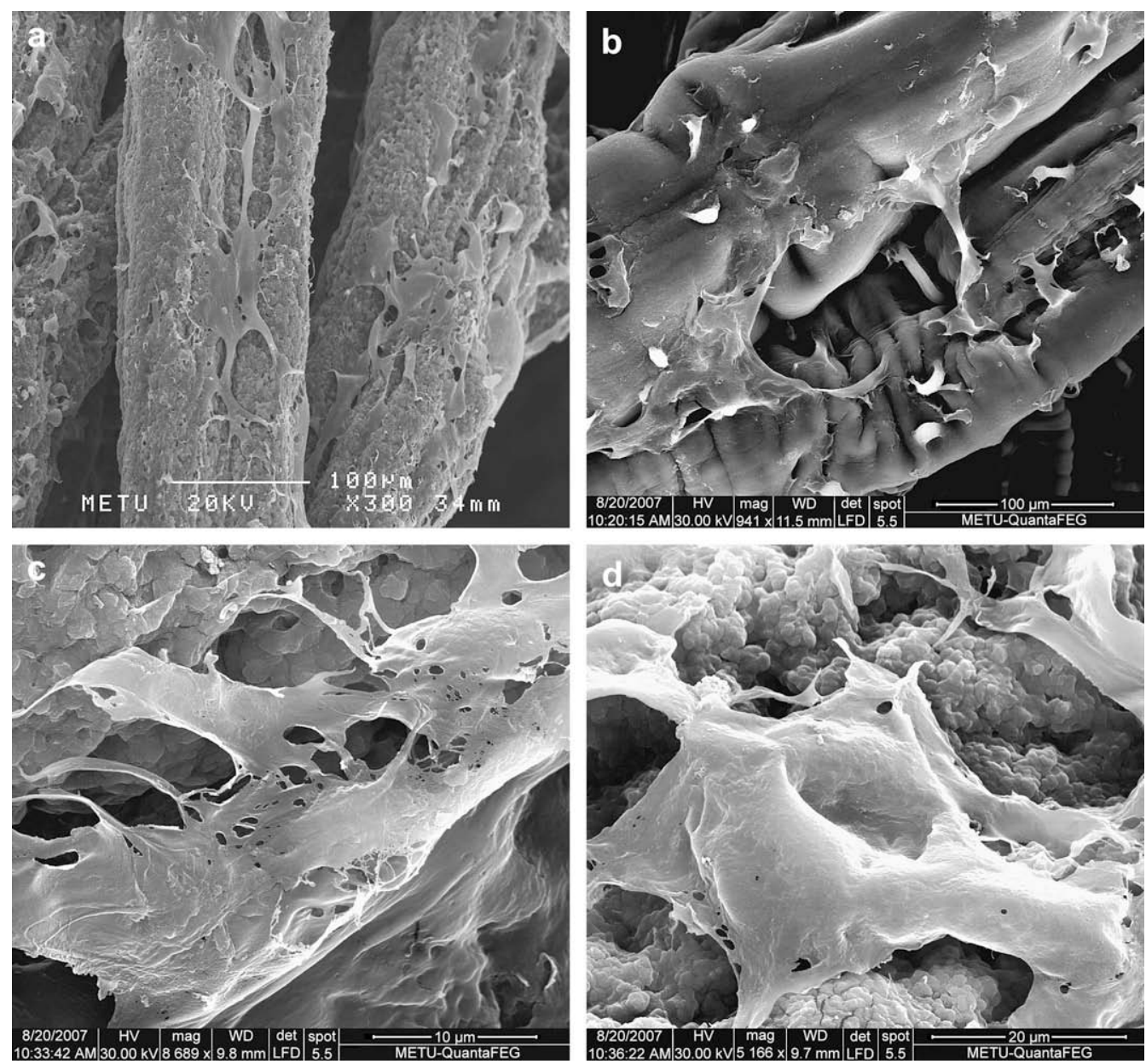

Fig. 7. MSC attachment and spreading on (a,b) CHI4-HAc2; (c,d) CHI4-PEO2-HAc5 scaffolds after 21 days of incubation.

only CHI4-HAc2 scaffolds were used with smooth fiber surfaces to properly demonstrate incorporation of the particles onto the fiber surfaces.

The release of BSA, a model protein, from the particle incorporated (NP-ON) and (NP-IN) constructs (CHI4-HAc2) was studied and compared with that of free nanoparticles. It was observed that although not influencing the overall release pattern, incorporation into the scaffolds (onto the fibers, NP-ON) suppressed the burst release in addition to slowing down the release for the rest of the period for both PLGA and PHBV nanocapsules (Fig. 10). The main difference in the release rates for both nanoparticles was observed during the first 3 days of the incubation where suppression in BSA release rates was observed. As described previously, PLGA nanocapsules, either free or incorporated in the scaffold structure, released their contents faster than PHBV counterparts which is why they were selected to serve as the early stage release component of the sequential delivery system. While the protein encapsulated in PLGA nanocapsules was almost completely released, both the free and the incorporated PHBV nanocapsules did not release their total content in the 21 days of the test. On the other hand, PLGA particles
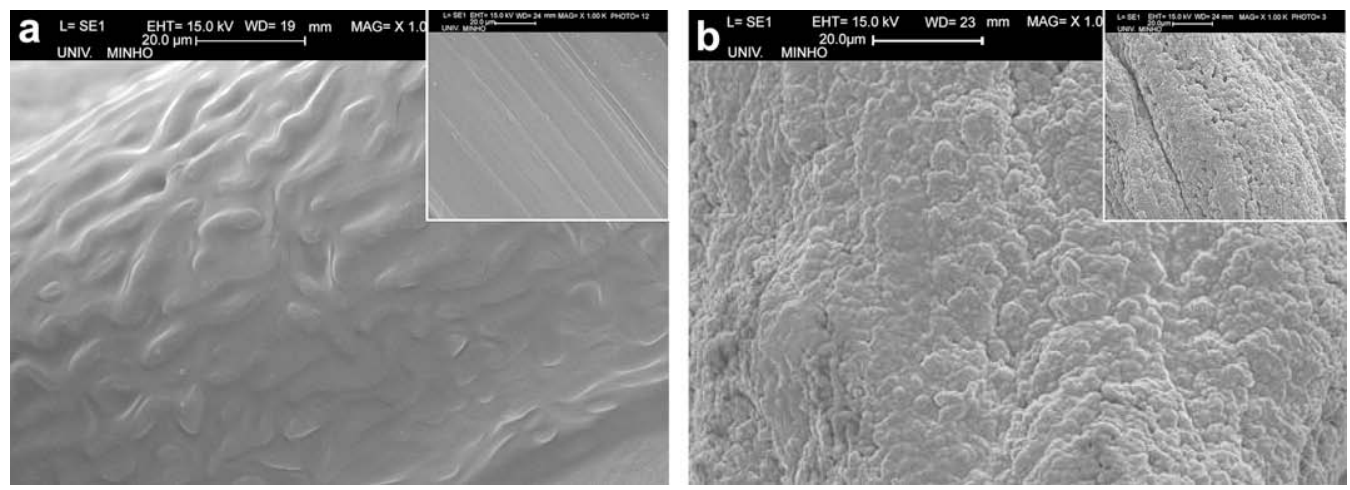

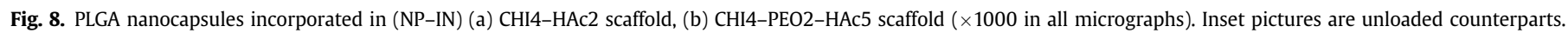



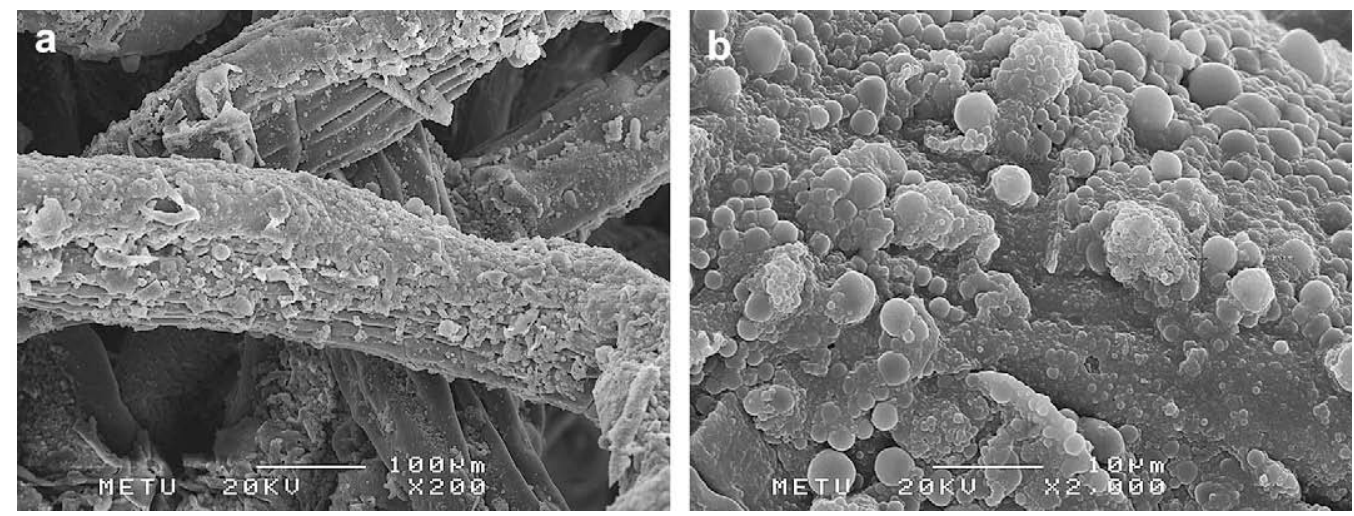

Fig. 9. Incorporation of PLGA nanocapsules onto CHI4-HAc2 scaffold (NP-ON), (a) $\times 200$, (b) $\times 2000$.

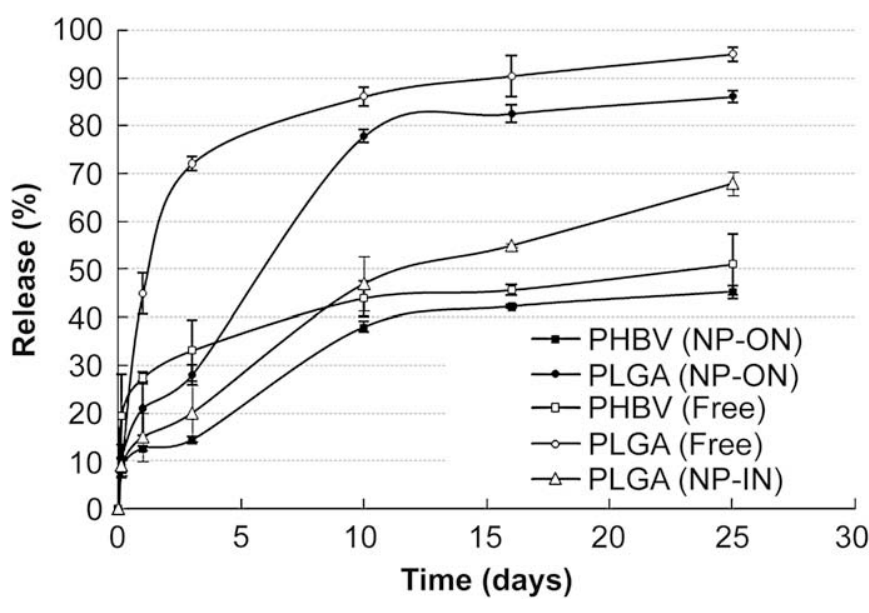

Fig. 10. Release of BSA from free and incorporated particles. incorporated in the fibers of CHI4-HAc2 scaffolds (NP-IN) revealed a much slower release rate, even slower than with NP-ON. It was observed that at the end of the incubation period of 25 days only $70 \%$ of the content was released from the NP-IN construct. The release of the content apparently is affected by the location of the drug carrying nanoparticles. When they are on the fiber there is only the diffusional restriction due the tortuosity of the path of the drug due to the fibers of the scaffold. When in the fiber, there is an additional diffusion restriction due to the drug traversing the fiber thickness.

The advantage of using nano-size particles and the incorporation approach (NP-IN and NP-ON) used in the delivery of BMP over use of free molecules is that the release from the capsules is controlled by the capsule wall properties. Especially in the NP-ON case, the release could be fine-tuned easily by changing the nanoparticle properties. When the incorporation within the fibers (NP-IN) is considered, it is deduced that the use of chitosan as the scaffold material is advantageous because it can swell (up to $600 \%$, Table 2) and the release from the nanocapsules probably takes place first into the fiber and then out the fiber into the release medium further prolonging the release of the molecules from the fibrous scaffold.

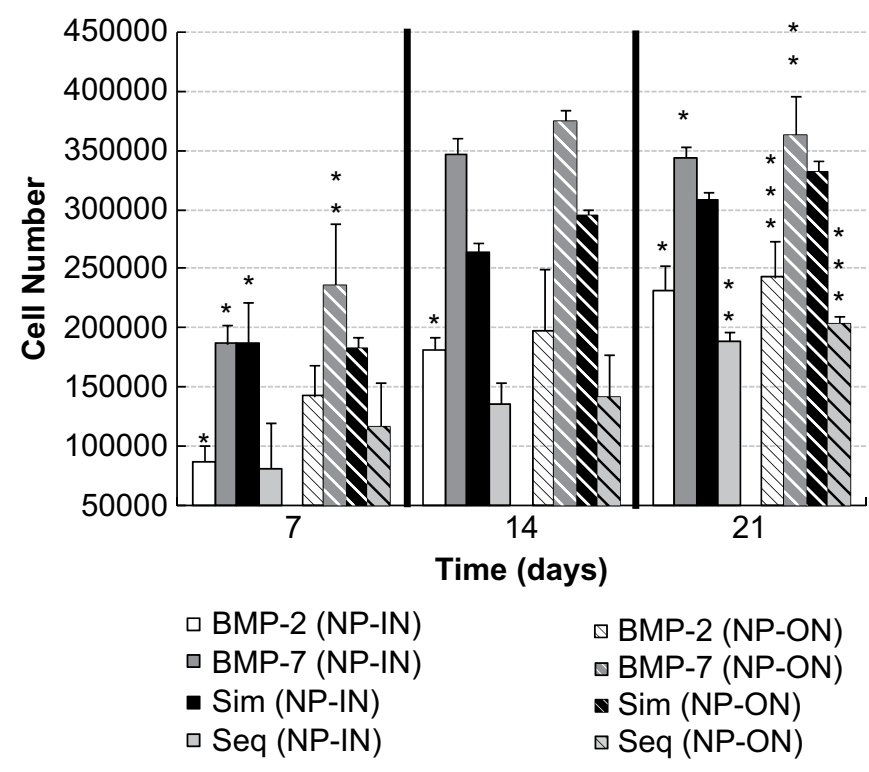

Fig. 11. Cell proliferation on BMP loaded particle incorporated chitosan fiber mesh scaffolds. $\left(n=3,{ }^{*} p<0.001,{ }^{* *} p<0.01\right.$, $\left.{ }^{* * *} p \leq 0.05\right)$. 


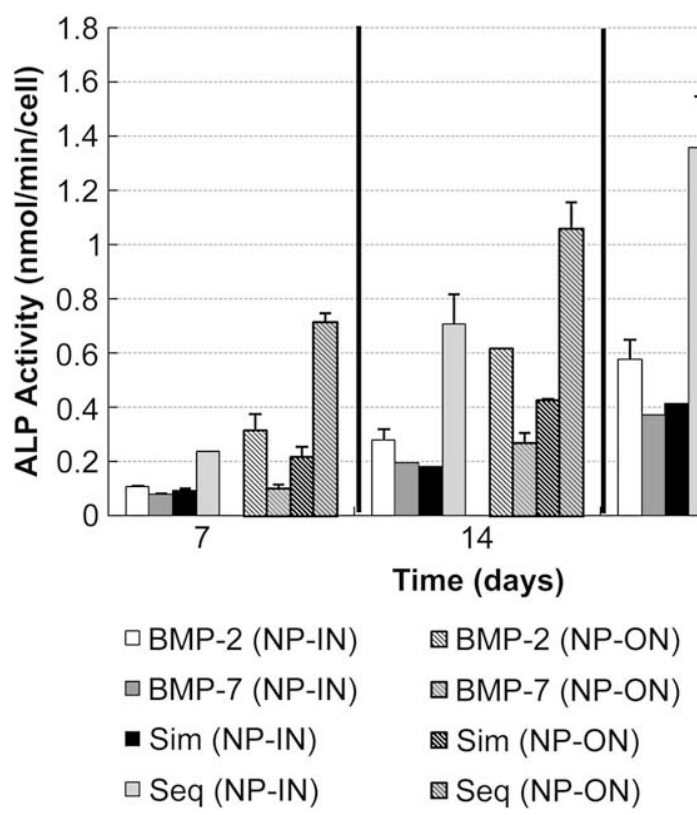

Fig. 12. Specific ALP Activity on BMP loaded particle incorporated chitosan fiber mesh scaffolds.

\subsection{Influence of sequential BMP-2/BMP-7 delivery from constructs on MSC proliferation and differentiation}

The effect of single, simultaneous, and sequential delivery of BMP-2 and BMP-7 released from constructs was investigated in vitro using rat bone marrow MSCs with two different incorporation methods. However, only one type of scaffold (CHI4-HAc2), with a smooth fiber surface and a higher initial MSC differentiation rate (Fig. 6), was used for the incorporation of BMP loaded nanoparticle incorporation studies. The simultaneous delivery of two growth factors was achieved by encapsulating both growth factors in PLGA nanocapsules. Therefore, in this condition both BMP-2 and BMP-7 were released to the medium in the beginning of the incubation. The difference observed in cell proliferation and differentiation through different administration routes of BMPs suggested that the growth factors were released in a bioactive form from the constructs for an extended period.

For every condition tested, particles incorporated on the fiber surfaces (NP-ON) led to higher cell numbers compared to particle incorporation within the fibers (NP-IN) indicating higher concentration due to higher rate of release of the growth factors (Fig. 11). The change in cell proliferation was significant at all times for all conditions ( $p \leq 0.05$, details given on the figure). Moreover, BMP-7 increased cell proliferation more than BMP-2 but had the lowest differentiation. Simultaneous delivery led to second highest proliferation rate. Sequential delivery had the lowest cell proliferation, but the highest ALP activity (Fig. 12) indicating higher cellular differentiation in case of sequential delivery of BMP-2 and BMP-7. The differences between ALP activity results were statistically significant at all time points for all conditions, as well $(p<0.001)$. Simultaneous delivery of two growth factors, on the other hand, failed to give high ALP activity results basically because proliferation was the stimulated biological mechanism rather than differentiation.

Cell number on chitosan fiber mesh scaffolds without loading with any BMPs (CHI4-HAc2) on day 7, 14 and 21 were $3.7^{*} 10^{5} \pm$ $9^{*} 10^{4}, 4.2^{*} 10^{5} \pm 7^{*} 10^{4}$ and $5.4^{*} 10^{5} \pm 1 * 10^{5}$ respectively (Fig. 5), were higher compared to the scaffolds loaded with the BMPs and this indicates the suppression of cell proliferation in the presence of BMPs regardless of the delivery condition.

\section{Conclusion}

In the present study, two bone growth factors, BMP-2 and BMP-7, were encapsulated in PLGA and PHBV nanocapsules which were then incorporated into fibrous chitosan scaffolds. The system constructed by the incorporation of two populations of nanocapsules to deliver the BMPs in a sequential manner performed better in inducing differentiation (ALP activity) than individual nanocapsule populations or the populations designed to provide simultaneous release of the BMPs. Of the sequential delivery systems, the one with the nanocapsules attached on the fibers performed better than the one where the nanocapsules were embedded in the fiber structure. It can be stated that sequential growth factor delivery is a better approach than individual growth factor use for tissue engineering due to its mimicking the natural process of healing.

\section{Acknowledgements}

This project was conducted within the scope of the EU FP6 NoE Project Expertissues (NMP3-CT-2004-500283). We acknowledge the support to PY through the same project in the form of an integrated PhD grant. We also would like to acknowledge the support from Scientific and Technical Research Council of Turkey (TUBITAK) through project METUNANOBIOMAT (TBAG 105T508).

\section{References}

[1] Stevens B, Yang Y, Mohandas A, Stucker B, Nguyen KT. A review of materials fabrication methods, and strategies used to enhance bone regeneration in engineered bone tissues. J Biomed Mater Res B Appl Biomater 2008;85B:573-82.

[2] Kose GT, Kenar H, Hasirci N, Hasirci V. Bone generation on PHBV matrices: an in vitro study. Biomaterials 2003;24:1949-58.

[3] Tuzlakoglu K, Alves CM, Mano CF, Reis RL. Production and characterization of chitosan fibers and 3-D fiber mesh scaffolds for tissue engineering applications. Macromol Biosci 2004;4:811-9.

[4] Ndreu A, Nikkola L, Ylikauppilar H, Ashammakhi N, Hasirci V. Electrospun biodegradable nanofibrous mats for tissue engineering. Nanomed 2008;3:45-60.

[5] Tuzlakoglu K, Bolgen N, Salgado AJ, Gomes ME, Piskin E, Reis RL. Nanoand micro-fiber combined scaffolds: a new architecture for bone tissue engineering. J Mater Sci Mater Med 2005;16:1099-104.

[6] Yilgor P, Sousa RA, Reis RL, Hasirci N, Hasirci V. 3D Plotted PCL scaffolds for stem cell based bone tissue engineering. Macromol Symp 2008;269:92-9.

[7] Wiria FE, Leong KF, Chua CK, Liu Y. Poly- $\varepsilon$-caprolactone/hydroxyapatite for tissue engineering scaffold fabrication via selective laser sintering. Acta Biomater 2007;3:1-12.

[8] Lee JY, Nam SH, Im SY, Park YJ, Lee YM, Seol YJ, et al. Enhanced bone formation by controlled growth factor delivery from chitosan-based biomaterials. J Control Release 2002;78:187-97.

[9] Edlund U, Danmark S, Albertsson AC. A strategy for the covalent functionalization of resorbable polymers with heparin and osteoinductive growth factor. Biomacromolecules 2008;9:901-5.

[10] Ziegler J, Anger D, Krummenauer F, Breitig D, Fickert S, Guenther KP. Biological activity of recombinant human growth factors released from biocompatible bone implants. J Biomed Mater Res 2008;86A:89-97.

[11] Shen YH, Shoichet MS, Radisic M. Vascular endothelial growth factor immobilized in collagen scaffold promotes penetration and proliferation of endothelial cells. Acta Biomater 2008;4:477-89.

[12] Lee SH, Shin H. Matrices and scaffolds for delivery of bioactive molecules in bone and cartilage tissue engineering. Adv Drug Deliv Rev 2007;59:339-59.

[13] Urist MR. Bone: formation by autoinduction. Science 1965;150:893-9.

[14] Bessa PC, Casal M, Reis RL. Bone morphogenetic proteins in tissue engineering: the road from the laboratory to the clinic, part I (basic concepts). J Tissue Eng Regen Med 2008;2:1-13.

[15] White AP, Vaccaro AR, Hall JA, Whang PG, Friel BC, McKee MD. Clinica applications of BMP-7/OP-1 in fractures, non-unions and spinal fusion. Int Orthop 2007;31:735-41.

[16] McKay WF, Peckham SM, Badura JM. A comprehensive clinical review of recombinant human bone morphogenetic protein-2 (INFUSE ${ }^{\circledR}$ bone graft). Int Orthop 2007;31:729-34.

[17] Cho TJ, Gerstenfeld LC, Einhorn TA. Differential temporal expression of members of the transforming growth factor beta superfamily during murine fracture healing. J Bone Miner Res 2002;17:513-20.

[18] Hsu HP, Zanella JM, Peckham SM, Spector M. Comparing ectopic bone growth induced by rhBMP-2 on an absorbable collagen sponge in rat and rabbit models. J Orthop Res 2006;24:1660-9. 
[19] Jansen JA, Vehof JWM, Ruhe PQ, Deutman HK, Kuboki Y, Takita H, et al. Growth factor-loaded scaffolds for bone engineering. J Control Release 2005; 101:127-36.

[20] Keskin DS, Tezcaner A, Korkusuz P, Korkusuz F, Hasirci V. Collagen-chondroitin sulfate-based PLLA-SAIB-coated rhBMP-2 delivery system for bone repair. Biomaterials 2005;26:4023-34.

[21] Simmons CA, Alsberg E, Hsiong S, Kim WJ, Mooney DJ. Dual growth factor delivery and controlled scaffold degradation enhance in vivo bone formation by transplanted bone marrow stromal cells. Bone 2004:35:562-9.

[22] Patel ZS, Young S, Tabata Y, Jansen JA, Wong MEK, Mikos AG. Dual delivery of an angiogenic and an osteogenic growth factor for bone regeneration in a critical size defect model. Bone 2008;43:931-40.
[23] Raiche AT, Puleo DA. In vitro effects of combined and sequential delivery of two bone growth factors. Biomaterials 2004;25:677-85.

[24] Basmanav FB, Kose GT, Hasirci V. Sequential growth factor delivery from complexed microspheres for bone tissue engineering. Biomaterials 2008;29:4195-204.

[25] Yilgor P, Hasirci N, Hasirci V. Sequential BMP-2/BMP-7 delivery from polyester nanocapsules. J Biomed Mater Res Part A, in press.

[26] Gomes ME, Holtorf HL, Reis RL, Mikos AG. Influence of the porosity of starchbased fiber mesh scaffolds on the proliferation and osteogenic differentiation of bone marrow stromal cells cultured in a flow perfusion bioreactor. Tissue Eng 2006;12:801-9.

[27] Kenar H, Kose GT, Hasirci V. Tissue engineering of bone on micropatterned biodegradable polyester films. Biomaterials 2006;27:885-95. 